\title{
Remote Sensing of the Earth for Agroecosystem Monitoring, Crop Production Planning and Management
}

\author{
Victor P. Yakushev \\ Agrophysical Research Institute, Saint-Petersburg, Russia \\ E-mail:vyakushev@agrophys.ru
}

\begin{abstract}
The paper presents the advanced possibilities and prospects of implementation of aerospace methods and information support technologies in agriculture discussed on the Second All-Russian Scientific Conference with international participation "The Use of Remote Sensing of the Earth in Agriculture" conducted at Agrophysical Research Institute on September 26-28th, 2018. A constructive exchange of views on the use of new approaches, models and hardware-software tools for remote sensing data interpretation has taken place. The significant role of the data obtained with unmanned aerial vehicles for nitrogen and water state management of crop canopy and planning agromeliorative measures was highlighted. Particular attention was addressed to the problem of the remote sensing data application in precision agriculture and necessity to organize a network of specialized test polygons for the development of techniques and methods of satellite agromonitoring.
\end{abstract}

Keywords: remote sensing of the Earth, agroecosystem monitoring, precision agriculture, mathematical models, ameliorative systems, in-field heterogeneity, decision support systems, field experiment management, test polygons. 


\title{
ДИСТАНЦИОННОЕ ЗОНДИРОВАНИЕ ЗЕМЛИ В ЗАДАЧАХ МОНИТОРИНГА АГРОЭКОСИСТЕМ, ПЛАНИРОВАНИЯ И УПРАВЛЕНИЯ ПРОИЗВОДСТВОМ РАСТЕНИЕВОДЧЕСКОЙ ПРОДУКЦИИ
}

\author{
В.П. Якушев \\ ФГБНУ «Агрофизический научно-исследовательский институт» (АФИ), Санкт-Петербург, \\ Россия \\ E-mail:vyakushev@agrophys.ru
}

\begin{abstract}
На прошедшей в АФИ 26-28 сентября 2018 г. II Всероссийской научной конференции с международным участием «Применение средств дистанционного зондирования Земли в сельском хозяйстве» широко обсуждались современные возможности, тенденции и перспективы развития и использования аэрокосмических методов и технологий информационного обеспечения сельского хозяйства. Состоялся конструктивный обмен мнениями по вопросам применения подходов, моделей и программно-аппаратных средств, предназначенных для совершенствования технологий интерпретации спутниковых данных. Отмечена значимая роль использования данных, получаемых с помощью беспилотников, в задачах планирования агромелиоративных мероприятий и оперативного управления азотным и водным режимами посевов. Особое внимание уделено проблематике применения ДЗЗ в точном земледелии и целесообразности создания сети специализированных полигонов для совершенствования и развития методов космического агромониторинга.
\end{abstract}

Ключевые слова: дистанционное зондирование Земли, мониторинг агроэкосистем, точное земледелие, математические модели, мелиоративные системы, внутриполевая неоднородность, системы управления, опытное дело, тестовые полигоны.

Эффективное решение задачи перехода к цифровым технологиям «умного сельского хозяйства» возможно только на основе создания и внедрения информационных технологий, включая технологии информационного обеспечения сельскохозяйственного производства. Традиционная практика получения, обработки и представления данных не соответствует требуемому уровню как по полноте пространственного распределения информации, так и по ее достоверности. Наиболее перспективным и масштабируемым ресурсом информационного обеспечения сельскохозяйственного производства являются методы и средства дистанционного зондирования Земли (ДЗ3).

Применение спутниковых данных Д3З в сельском хозяйстве начиналось с простейших методов, основанных на использовании вегетационных и других индексов, представляющих собой комбинации фиксируемых величин отражения в различных спектрах электромагнитного излучения, как правило, оптического диапазона. Еще на первой конференции по дистанционному зондированию подчеркивалась ограниченность использования данного подхода для решения задач управления продукционным процессом сельскохозяйственных культур, а также отмечен его основной недостаток отсутствие надлежащего доступа к соответствующей спутниковой информации [1].

К настоящему времени благодаря разработкам ученых ИКИ РАН и созданию ЦКП «ИКИ-мониторинг» спутниковые технологии предоставляют пользователям возможности не только поиска и получения объективной первичной информации, но и ее обработки, а также анализа состояния агроэкосистем с использованием распределенных вычислительных ресурсов. Данная возможность обусловлена следующими основными факторами:

- существенным повышением качества, частоты получения и доступности спутниковой информации. По состоянию на начало 2018 г. на орбите Земли действовало 
более 420 (по данным http://database.eohandbook.com) космических аппаратов Д33, несколько десятков из которых предоставляют общедоступные данные;

- стремительным развитием новых методов и технологий обработки спутниковых данных (в том числе полностью автоматизированных). Например, в последние годы был разработан целый ряд подобных методов, в том числе:

1. методы картографирования основных типов сельскохозяйственных культур $[2,3]$;

2. методы оценки используемых сельскохозяйственных земель $[4,5,6]$;

3. методы оценки состояния сельскохозяйственных культур $[7,8]$.

- появлением новых технологий и возможностей построения специализированных систем дистанционного мониторинга $[9,10]$.

Наряду с возможностями ЦКП «ИКИ-мониторинг», позволяющей разрабатывать, внедрять и поддерживать различные информационные системы дистанционного мониторинга сельскохозяйственных земель и посевов, рассматривались и другие оригинальные подходы, а также результаты сопряженного использования спутниковых данных, математических моделей и опорной информации. Большой интерес у участников конференции вызвали, например, доклады, в которых:

- освещены этапы развития методов применения данных дистанционного зондирования Земли в сельском хозяйстве: от простейших подходов, основанных на индексах различного рода, до решения задач управления в системах точного земледелия. Информационной базой указанных задач являются оценки параметров состояния посевов и почвенной среды, получаемые на основе данных дистанционного зондирования и комплекса математических моделей. В состав такого комплекса входят модели оптических измерений и модели динамики параметров состояния посевов и почвенной среды. Использование таких моделей позволяет за счет комплексирования информации оценивать по данным дистанционного зондирования параметры состояния посевов и почвенной среды, необходимые для решения задач управления в системах точного земледелия [11];

- представлены оценки характеристик водного и теплового режимов участков территории Саратовского и Волгоградского Заволжья, занятых посевами сельскохозяйственных культур, для вегетационного сезона 2012 г. Оценки получены при помощи моделей вертикального влаго- и теплообмена поверхности суши с атмосферой SVAT и SWAP и модели SEBS с использованием спутниковых данных о состоянии подстилающей поверхности и метеорологических условиях, а также аддитивной модели водоудерживания для текстурных почв [12];

- изучены пространственные корреляции станционных данных измерений запасов продуктивной влаги в пахотном и 10-сантиметровом слоях почвы и спутниковых данных об относительной влажности верхнего слоя почвы (ИСЗ MetOp-А и В, скаттерометр ASCAT) по европейской территории России. Для этого в тестовом режиме использовалась версия компьютерной технологии, при помощи которой осуществлялся контроль станционных данных, а затем выполнялся объективный анализ [13].

Значительная часть выступлений на конференции была посвящена применению беспилотных летательных аппаратов (БЛА). Их роль особенно значима в оценке состояния мелиоративных систем и исследовательских работах по изучению процессов роста и механизмов управления продуктивностью различных сельскохозяйственных культур, возделываемых с применением элементов технологии точного земледелия.

Состояние объектов мелиоративной инфраструктуры влияет на эффективность управления использованием сельскохозяйственных мелиорируемых земель и представляет собой серьезную экологическую проблему в случае их деградации. Одними из ключевых факторов деградационных процессов являются упадок и неэффективное использование существующих дренажных систем. Выделены основные этапы обработки данных дистанционного зондирования, полученных с помощью автоматизированного 
беспилотного летательного аппарата (квадрокоптер Геоскан 401), которые используются для решения следующих задач: определение расположения закрытой дренажной системы сельскохозяйственного поля и локализация ее неисправностей; оценка степени зарастания открытой дренажной системы; фиксирование границ участков поля, подверженных водной эрозии. Представлены конкретные примеры решения указанных задач, а также методики использования данных аэрофотосъемки для составления ортофотопланов, привязанных к глобальной системе позиционирования, формирования моделей рельефа местности, моделей водных потоков на сельскохозяйственных полях и расчета основных вегетационных индексов $[14,15]$.

На секционных заседаниях проходили содержательные дискуссии по вопросам, связанным с применением БЛА, а также наземных мобильных и стационарных измерительных систем для получения, калибровки, комплексирования и последующего сопряженного анализа разнородной информации. Анализ полученной информации позволяет в динамике выявлять внутриполевую неоднородность показателей продуктивности посевов и плодородия почв. Такой подход позволил развернуть комплексные многолетние исследования. Появилась возможность изучить влияние сложности и контрастности почвенного покрова на продуктивность различных сельскохозяйственных культур, возделываемых с применением информационных технологий точного земледелия [16]. Так, например, с целью изучения влияния на продукционный процесс яровой пшеницы различных агротехнологий на фоне естественной неоднородности полей севооборота на полигоне АФИ проводился многолетний оригинальный сравнительный эксперимент. Изучалось несколько вариантов технологий различной интенсивности: экстенсивная технология - контрольный вариант, при котором проводятся только основные агротехнические мероприятия без дополнительных затрат; высокоинтенсивная технология, предусматривающая внесение удобрений и средств защиты растений в дозах, полностью удовлетворяющих потребность посева в период вегетации; точное земледелие, отличительной особенностью которого является дифференцированный подход к расчёту и внесению средств химизации в зависимости от показателей плодородия полей и состояния посевов. Сравнительный анализ показал, что в результате дифференцированного внесения азотных удобрений по технологии ТЗ урожайность повысилась на $29 \%$, а их экономия составила $25,9 \%$ в среднем за 7 лет [17]. При этом сокращение объёма применяемых азотных удобрений в варианте "точное земледелие" было достигнуто в основном за счёт дифференцированных подкормок в вегетационный период. Для проведения азотных подкормок на опытных полях закладывались тестовые площадки, в почву которых вносился азот в различных дозах. Регулярно проводилось дистанционное обследование посевов с помощью беспилотных летательных аппаратов самолётного и вертолётного типов, разработанных инженерами АФИ [18]. На них размещались цифровые камеры для получения аэрофотографий посевов в видимой и инфракрасной областях спектра электромагнитного излучения. На каждой аэрофотографии поля отображались тестовые площадки с известной дозой внесённых азотных удобрений. Средние значения колориметрических (цветовых) характеристик посева на каждой из тестовых площадок являются эталонными. Посредством сравнения с ними характеристик остальных зон поля проводится оценка потребности растений в азоте. В частности, выделение участков поля, на которых необходимо осуществить внесение азотных удобрений, выполнялось с помощью разработанного специалистами АФИ автоматизированного метода построения калибровочных кривых по колориметрическим характеристикам аэрофотографий посевов [19]. Такой способ позволяет провести оценку обеспеченности растений азотом на любом участке поля и является доступным, недорогостоящим и достаточно точным методом мониторинга посевов, оценки их потребности в азотном питании и прецизионного внесения удобрений. 
Целесообразность более широкого применения технологий точного земледелия (T3) в производстве растениеводческой продукции в настоящее время не вызывает сомнений. Однако эффективность перехода от традиционной агрономии к информационным технологиям ТЗ в каждом конкретном случае может быть различной и должна быть оценена заранее. Принципиальное значение в данном вопросе имеет следующее обстоятельство. Традиционные приемы обычно предусматривают дифференциацию норм технологического воздействия от поля к полю, т.е. пространственная вариабельность условий формирования урожая учитывается только в пределах достаточной обширной сельскохозяйственной территории, превышающей размеры отдельных полей. В ТЗ технологические воздействия дифференцируются в масштабах отдельных полей. Отсюда следует, что переход к технологиям Т3 на конкретном поле может быть оправдан лишь в тех случаях, когда внутриполевое варьирование условий формирования урожая оказывает существенное влияние на общую картину неоднородности рассматриваемой сельскохозяйственной территории по тем же факторам урожайности. Следовательно, объективно существующая вариабельность физиологического состояния посевов и окружающей среды, наличие латентных факторов, невозможность точного предсказания метеорологических условий, а также трудности учета других факторов, оказывающих значимое воздействие на агроценоз, приводят к необходимости разработки новых количественных подходов и методов обнаружения внутриполевой изменчивости условий формирования урожая [16].

Создание новых инструментов для выделения границ внутриполевой неоднородности базируется на расширении возможностей использования спутниковых данных ДЗ3. Учёными АФИ предложен базовый алгоритм обнаружения и выделения границ внутриполевой изменчивости по гиперспектральным снимкам и оптическим критериям. Алгоритм рассмотрен на примере дефицита воды или (и) азота. Дефицит воды и ее неравномерное распределение по полю могут значительно исказить результаты дистанционной оценки потребности растений в удобрении. Предварительно было изучено изменение оптических характеристик растений при дефиците азота и воды. Исследования проводились в контролируемых условиях с целью исключения воздействия на растения других неблагоприятных факторов среды [20]. В результате проведенных исследований предложены конкретный перечень оптических критериев (индексов отражения) и набор количественных характеристик по каждому критерию для оценки физиологического состояния яровой пшеницы при оптимальных условиях и дефиците азота и воды. Существенные различия оптических характеристик растений, испытывающих дефицит азота и воды, являются надежным обоснованием реализуемости алгоритма интерпретации (дешифрирования) спутниковых данных Д33. Наличие подобной информации открывает значительные перспективы для автоматизации процесса дешифрирования спутниковых гиперспектральных снимков с целью выделения границ внутриполевой неоднородности. Реализация соответствующего алгоритма позволит не только обнаружить участок посева с угнетенными растениями, но и выявить стрессор, действие которого стало причиной угнетения [21].

Для дальнейшего развития методов и средств Д3З в интересах сельского хозяйства необходимы новые подходы к совершенствованию опытного дела и разработке современного методологического, физико-технического и технологического обеспечения для проведения экспериментов в различных почвенно-климатических зонах России. В связи с этим в Постановление конференции внесено предложение обратиться в Президиум РАН с просьбой рассмотреть и оказать содействие в исполнении Постановления Бюро отделения сельскохозяйственных наук РАН № 1 от 21 января 2016 г., принятие которого было инициировано АФИ и ИКИ, о создании сети специализированных тестовых полигонов для подспутниковых наблюдений на базе опытных полей научноисследовательских институтов зернопроизводящей зоны РФ и оснащении их специализированными информационно-измерительными автоматизированными 
системами для полевых наблюдений, а также испытания оборудования и апробации методик, предназначенных для оценки агроэкосистем и усовершенствования алгоритмов дешифрирования данных ДЗ3.

Создание сети тестовых полигонов позволит придать мощный импульс дальнейшему развитию и практическому применению методов дистанционного зондирования из космоса для решения широкого комплекса задач мониторинга сельского хозяйства. Опорная информация, регулярно собираемая на тестовых полигонах с использованием унифицированных методик, позволит создать метод обработки материалов дистанционного зондирования, обеспечивающий получение откалиброванных и верифицированных данных об использовании пахотных земель, площади и состоянии посевов различных культур, а также их прогнозной и фактической урожайности.

\section{References}

[1] The remote sensing of the Earth in agriculture. Materials of the All-Russian Scientific Conference, St. Petersburg, ARI, 2015, 196 p.

[2] Bartalev S.A., Egorov V.A., Zharko V.O., Loupian E.A., Plotnikov D.E., Hvostikov S.A., Shabanov N.V., Satellite mapping of vegetation cover in Russia. Moscow, SRI, 2017, 208 p.

[3] Plotnikov D.E., Bartalev S.A., Zharko V.O., Mikhailov V.V., Prosyannikova O.I., An experimental assessment of crop types recognisability using time-series of intra-seasonal spectral reflectance measurements by satellite sensor, Sovremennye Problemy Distantsionnogo Zondirovaniya Zemli iz Kosmosa, 2011, No. 8 (1), pp. 199-208.

[4] Bartalev S.A., Egorov V.A., Loupian E.A., Plotnikov D.E., Uvarov I.A., Recognition of arable land based on long-term satellite data of a MODIS spectroradiometer and locally adaptive classification, Komp'yuternaya optika. Samara, IPSI, 2011, Vol. 35, No. 1, pp. 103-116.

[5] Plotnikov D.E., Bartalev S.A., Loupian E.A., The recognition features to map arable lands based on multi-annual MODIS Earth observation data, Sovremennye Problemy Distantsionnogo Zondirovaniya Zemli iz Kosmosa, 2010, No. 7 (1), pp. 330-341.

[6] Bartalev S.A., Loupian E.A., Tolpin V.A., Elkina E.S., Web-based Earth Observation data analysis system VEGA-GEOGLAM in support of global agricultural monitoring research and developments, Geoinformatics Research Papers, 2017, Vol. 5, BS1002, pp. 166. DOI: 10.2205/CODATA2017.

[7] Tolpin V.A., Loupian E.A., Bartalev S.A., Plotnikov D.E., Matveev A.M., Possibilities of analyzing the state of agricultural vegetation using the VEGA satellite service, Optika atmosfery i okeana, 2014, Vol. 27, No. 7 (306), pp. 581-586.

[8] Tolpin V.A., Bartalev S.A., Burcev M.A., Efremov V.Yu., Loupian E.A., Mazurov A.A., Matveev A.M., Proshin A.A., Flitman E.V., Assessment of crop condition based on interannual dynamics using MODIS data, Sovremennye Problemy Distantsionnogo Zondirovaniya Zemli iz Kosmosa, 2007, No. 2, pp. 380-389.

[9] Loupian E.A., Balashov I.V., Burtsev M.A., Efremov V.Yu., Kashnickij A.V., Kobec D.A., Krasheninnikova Yu.S., Mazurov A.A., Nazirov R.R., Proshin A.A., Sychugov I.G., Tolpin V.A., Uvarov I.A., Flitman E.V., Development of information systems design technologies, Sovremennye Problemy Distantsionnogo Zondirovaniya Zemli iz Kosmosa, 2015, No. 12 (5), pp. 53-75.

[10] Loupian E.A., Burcev M.A., Proshin A.A., Kobec D.A., Evolution of remote monitoring information systems development concepts, Sovremennye Problemy Distantsionnogo Zondirovaniya Zemli iz Kosmosa, 2018, No. 15 (3), pp. 53-66. DOI: 10.21046/2070-7401-2018-15-3-53-66.

[11] Mikhailenko I.M., Development of methods and tools for the application of remote sensing data in agriculture, Tendencii razvitiya nauki $i$ obrazovaniya, 2018, Vol. 3, pp. 70-83. DOI: 10.25695/agrophysica.2018.2.18690. 
[12] Zeiliger A.M., Muzylev E.L., Ermolaeva O.S., Startseva Z.P., Volkova E.V., The use of remote sensing data in modeling the water and thermal regimes of agrocenoses. Materialy II nauchnoj konferencii «Distancionnoe zondirovanie Zemli v sel'skom hozyajstve», St. Petersburg, 2018, pp. 66-75. (http://www.agrophys.ru/).

[13] Bykov F.L., Vasilenko E.V., Gordin V.A., Tarasova L.L., Statistical structure of the field of humidity of the upper soil layer according to ground and satellite observations, Meteorologiya $i$ gidrologiya, 2017, No. 6, pp. 68-84.

[14] Petrushin A.F., Mitrofanov E.P., Assessment of the agricultural field drainage system status using remote sensing data, Vestnik rossijskoj sel'skohozyajstvennoj nauki, 2017, No. 4, pp. 17-20.

[15] Yakushev V.P., Lekomtsev P.V., Matveenko D.A., Petrushin A.F., Yakushev V.V., Application of distance pit testing in the system of precision agriculture, Vestnik rossijskoj sel'skohozyajstvennoj nauki, 2015, No. 1, pp. 23-25.

[16] Yakushev V.P., Yakushev V.V., Prospects of smart agriculture in Russia, Vestnik RAN, 2018, Vol. 88, No. 9, pp. 773-784. DOI: 10.31857/S086958730001690-7.

[17] Yakushev V.P., Lekomtsev P.V., Voropaev V.V., Konev A.V., Pervak T.S., Discriminatory application of the chemicals under the spring wheat cultivation, Vestnik rossijskoj sel'skohozyajstvennoj nauk, 2017, No. 4, pp. 13-17.

[18] Yakushev V.P., Yakushev V.V. Information support for precision farming. St. Petersburg, PIYAF $R A N, 2007,384 \mathrm{p}$.

[19] Mitrofanova O.A., Bure V.M., Kanash E.V., Math module to automate the colorimetric method for estimating nitrogen status of plants, Vestnik SPBGU, 2016, No. 1, pp. 87-93.

[20] Kanash E.V., Yakushev V.P., Osipov Y.A., Rusakov D.V., Blokhina S.Yu., Kravcova A.V., Optical characteristics of the spring wheat leaves under the nitrogen and water deficiency conditions, Vestnik rossijskoj sel'skohozyajstvennoj nauki, 2017, No. 4, pp. 13-17.

[21] Yakushev V., Kanash E., Rusakov D., Blokhina S., Specific and non-specific changes in optical characteristics of spring wheat leaves under nitrogen and water deficiency, Advances in Animal Biosciences: Precision Agriculture (ECPA 2017), 2017, 8:2, pp. 229-232. 